Aim of the study: The first aim was to investigate the knowledge and awareness of oncologists concerning febrile neutropenia (FN) risk assessment and indications for granulocyte colony-stimulating factor (G-CSF) primary prophylaxis (PP), based on current therapeutic guidelines (PTOK and EORTC). The second aim was to educate the oncologists on best practices for risk assessment and neutropenia management.

Material and methods: The project participants included 169 oncologists from 7 regions working in large specialist oncological centres, university hospitals, regional and city hospitals, specialist outpatient clinics, and oncological wards in small local hospitals. The participants completed a questionnaire based on seven prepared clinical cases of patients with different tumour types and patient characteristics, receiving chemotherapy (CT), and with different levels of FN risk. Participants answered questions related to FN risk assessment and $\mathrm{G}-\mathrm{CSF}$ use. After completing the questionnaire, the participants proceeded to an educational module in which they were provided with an analysis of correct diagnostic and therapeutic procedures according to the PTOK and EORTC guidelines.

Results and Conclusions: Febrile neutropenia risk assessment was found to be a routine procedure performed for over $90 \%$ of the clinical cases by the participant oncologists. However, the FN risk assessment of clinical cases was correct and consistent with therapeutic guidelines in only $65 \%$ of responses. Indications for G-CSF PP were properly identified in $76 \%$ of responses and it appeared that indications for G-CSF PP were more likely to be correctly identified in patients receiving high-risk or low-risk regimens than in those receiving intermediate-risk regimens, where the decision to give G-CSF PP is based on additional assessment of patient risk factors. The vast majority of participants who correctly identified the need for PP administered G-CSF in accordance with the dose and schedule recommended by PTOK and EORTC.

Key words: chemotherapy induced neutropenia, G-CSF, febrile neutropenia prophylaxis.

Contemp Oncol (Pozn) 2014; 18 (6): 419-424 DOI: $10.5114 /$ wo.2014.47904

\section{Clinical practice in febrile neutropenia risk assessment and granulocyte colony-stimulating factor primary prophylaxis of febrile neutropenia in Poland}

\author{
Marek Wojtukiewicz' ${ }^{1}$ Ewa Chmielowska², Emilia Filipczyk-Cisariz ${ }^{3}$, \\ Krzysztof Krzemieniecki ${ }^{4}$, Krzysztof Leśniewski-Kmak ${ }^{5}$, Maria M. Litwiniuk ${ }^{6}$, \\ Karolina Wieruszewska-Kowalczyk ${ }^{7}$, Elżbieta Kosno-Kruszewska ${ }^{7}$ \\ ${ }^{1}$ Department of Oncology, Medical University, Comprehensive Cancer Center, Bialystok, \\ Poland \\ ${ }^{2}$ Oncology Clinic, Oncology Centre, Bydgoszcz, Poland \\ ${ }^{3}$ Chemotherapy Department, Lower Silesian Oncology Centre, Wroclaw, Poland \\ ${ }^{4}$ Oncology Department, Jagiellonian University, Krakow, Poland \\ ${ }^{5}$ Department of Propedeutical Oncology, Medical University of Gdansk and Department \\ of Oncology and Radiotherapy, Gdynia Oncology Centre, Gdynia, Poland \\ ${ }^{6} \mathrm{Clinic}$ of Oncology, Poznan University of Medical Sciences, Poznan, Poland \\ ${ }^{7}$ Amgen Biotechnologia Sp. z o.o., Warsaw, Poland
}

\section{Introduction}

Febrile neutropenia (FN), described as a neutrophil count $<1000 / \mu$ l and a body temperature $>38.0^{\circ} \mathrm{C}[1]$, is a common complication in chemotherapy (CT) patients. The risk of FN depends on the chemotherapy regimen and also on the general condition of the patient, the presence of comorbidities, and the tumour stage. Prolonged severe neutropenia is the main risk factor for infections, which may increase the morbidity and mortality rate and lead to delayed administration or reduction of $\mathrm{CT}$ dose, possibly compromising clinical outcomes, especially in cases of intensive CT regimens [2]. According to the guidelines of the Polish Association of Clinical Oncology (PTOK) and the European Organisation for the Research and Treatment of Cancer (EORTC), FN risk assessment should be performed before each CT cycle and appropriate prophylaxis should be initiated [1]. Primary prophylaxis (PP) is recommended as a standard in CT regimens with a high FN risk (over 20\%). When the risk is moderate (between $10 \%$ and $20 \%$ ) patient-related factors should be taken into account. Primary prophylaxis is not recommended for CT regimens with a low FN risk (lower than 10\%) [3, 4]. Primary prophylaxis is defined as the administration of granulocyte colony-stimulating factors (G-CSF) from the first chemotherapy cycle. According to PTOK guidelines, a daily G-CSF (e.g. filgrastim) should be administered 24-72 hours after CT and continued until the expected nadir value is exceeded (usually 25-7 days) and a normal (or slightly lower, but stable) neutrophil count is achieved. A pegylated form of G-CSF, i.e. pegfilgrastim, is administered as a single injection for each CT cycle, given approximately 24 hours after CT.

This study provides a summary of the Polish project GoPractice: an analysis of clinical practice in the prophylaxis of FN carried out in 2012/2013 using an online educational format. The study had two objectives, cognitive and educational, including an assessment of the awareness of indications for primary and secondary prophylaxis based on the risk of FN, in accordance with the published diagnostic and therapeutic recommendations (PTOK and 
EORTC), and an analysis of diagnostic and therapeutic decisions made by the participating physicians based on seven clinical cases prepared by the experts involved in the project. The experts are the authors of the publication, representing seven Polish provinces in which the project was conducted.

\section{Material and methods}

The project participants were oncologists from the Polish provinces of Dolnoslaskie, Kujawsko-Pomorskie, Malopolskie, Podlaskie, Pomorskie, Slaskie, and Wielkopolskie. The participants worked in medical centres selected by the Experts, such as large specialised facilities (e.g. oncology centres and university hospitals), medium-sized hospitals with an oncological ward (e.g. specialised regional hospitals), city hospitals, and small medical centres (e.g. specialist clinics, non-public medical centres [NZOZ] and small hospitals with internal medicine wards and designated oncological beds). The project participants were oncologists with or without a specialisation and with various durations of clinical practice experience.

The participants were involved in a model analysis and decision-making exercise assessing their diagnostic and therapeutic decisions concerning indications and lack of indications for primary and secondary prophylaxis in seven clinical cases prepared by the experts. There were three cases of breast cancer, two of non-Hodgkin's lymphoma [diffuse large B-cell lymphoma (DLBCL) and primary mediastinal B-cell lymphoma (PMBL)], one of ovarian cancer, and one of non-small cell lung cancer (NSCLC). In all cases, the patients were treated with various chemotherapy regimens for which the FN risk has been characterised in the PTOK and EORTC guidelines. Based on their best knowledge, the project participants answered questions regarding FN risk assessment standards. Participants answered questions in four different categories:

In the Risk Assessment category, the participants answered the question whether they would assess FN risk for each of the cases in their routine clinical practice, based on their personal experience.

When answering the Risk Values question, the participants assessed the value of FN risk based on the chemotherapy regimen only. Participants were asked to assess clinical cases and give their estimate of the level of FN risk of the CT regimen.

When asked about Primary Prophylaxis, the participants had to identify the presence or absence of indications for primary prophylaxis with G-CSF. Participants were asked to assess clinical cases and determine whether the patient qualified for G-CSF PP based on the combination of FN risk from the $\mathrm{CT}$ regimen and individual patient factors.

The question concerning the Primary Prophylaxis Method required a description of G-CSF administration after the first cycle of CT in individual cases. Based on their estimate of the level of FN risk of the $\mathrm{CT}$ regimen, participants selected the choice of G-CSF type and the method of administration and dosing.

Based on the guidelines and Expert opinion, the FN risk of $\mathrm{CT}$ regimens was considered high for breast can- cer patients receiving AT (doxorubicin, docetaxel) and TAC (docetaxel, doxorubicin, cyclophosphamide); moderate for NHL patients receiving R-CHOP-21 (rituximab, cyclophosphamide, doxorubicin, vincristine, prednisone) and breast cancer patients receiving AC (doxorubicin, cyclophosphamide); and low for ovarian cancer patients receiving AC, NSCLC patients receiving VP (vinorelbine, cisplatin), and breast cancer patients receiving paclitaxel + trastuzumab or palliative treatment. The experts assessed that there is an indication to give G-CSF PP always when the CT regimen is mentioned as high FN risk in the PTOK and EORTC guidelines. When the $\mathrm{CT}$ regimen was associated with moderate risk of FN, the experts assessed that there is an indication to give G-CSF PP in patients treated with the intention of curing or prolonging life, and considered existing patient risk factors that increase the risk of FN. In their opinion, G-CSF PP was indicated in patients with DLBCL and $P M B L$ receiving $\mathrm{R}-\mathrm{CHOP}-21$, due to treatment intent and additional patient risk factors.

The experts assessed that there is no indication for G-CSF PP in the case of breast cancer patients receiving AC, which was considered as a moderate-risk regimen with no patient-related risk factors.

After completing the survey, the participants could review the educational part containing the correct answers, with explanations provided by the experts based on the PTOK and EORTC guidelines and their experience.

One hundred and eighty-five physicians were invited to participate in the project and 169 of them completed the survey. The educational module was completed by 138 physicians (82\%).

\section{Results}

When responses to all questions were combined, 75\% were answered correctly in the survey. The highest proportion of correct replies was given for DLBCL (80\%) and the lowest for palliative breast cancer (67\%). The percentage of correct answers was independent of the physicians' education level (specialisation vs. lack of specialisation), years of experience, type of health centre, or geographic location.

Indications or lack of indications for PP were correctly recognised in $76 \%$ of the responses. Approximately twothirds of the responses (65\%) followed the available treatment guidelines and correctly evaluated the FN risk of the $\mathrm{CT}$ regimen. These data are shown in Fig. 1.

\section{Febrile neutropenia risk assessment}

For over $90 \%$ of the respondents, FN risk assessment was a routine clinical procedure.

For over $90 \%$ of cases, the project participants declared that they would perform routine assessment of FN risk in their clinical practice. In clinical cases where there were indications for PP administration, 96\% of participants declared that they would routinely perform FN risk assessment if presented with such a case in their clinical practice; in cases where there were no indications for PP, $86 \%$ of participants would routinely perform an FN risk assessment. Although a high proportion of participants 


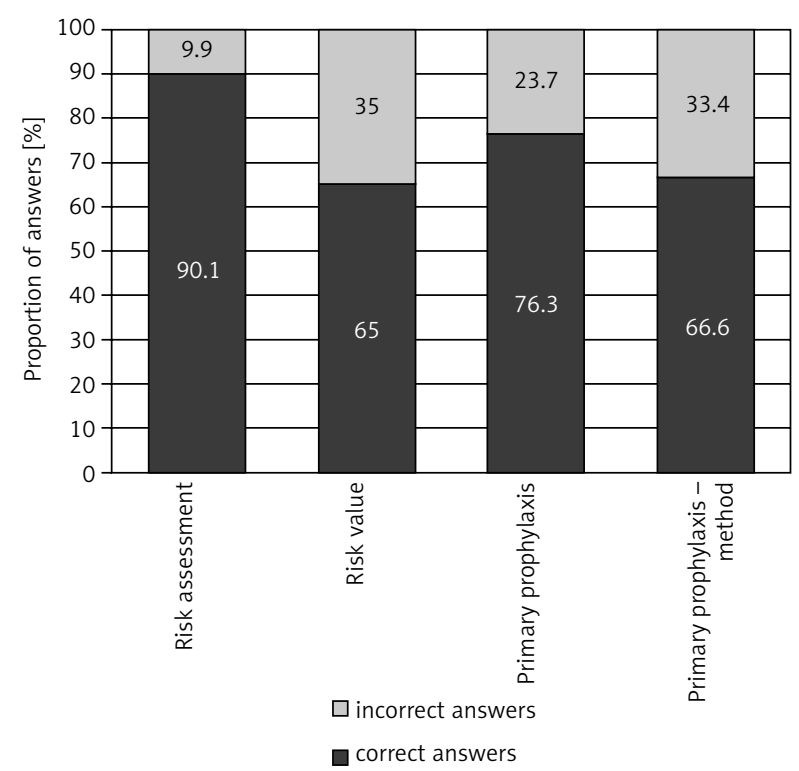

Fig. 1. The percentage of correct and incorrect answers by question category

considered FN risk assessment as routine in their clinical practice, the overall accuracy of risk assessment, based on the CT regimen risk, was only 65\% (Fig. 2).

\section{Assessment of febrile neutropenia risk based on the chemotherapy regimen}

In the case of chemotherapy regimens in which the FN risk was, according to PTOK and EORTC guidelines, greater than $20 \%$, the high FN risk was correctly identified in about $90 \%$ of responses. For regimens with a moderate risk of FN, over $70 \%$ of responses were in agreement with PTOK and EORTC recommendations. For low-risk regimens, half of the responses were consistent with the recommendations.

Among the participants who incorrectly assessed indications for PP (in the absence of indications), 82\% gave incorrect answers on the risk value for a specific $\mathrm{CT}$ regimen. When there were indications for PP, CT regimen FN risk was correctly evaluated in about $80 \%$ of responses; but in the absence of indications for PP, CT regimen FN risk was correctly evaluated in approximately $54 \%$ of responses. Thus, incorrect answers concerning the value of FN risk for a specific CT regimen were more frequent when there were no indications for PP.

\section{Assessment of indications for primary prophylaxis}

Indications or lack of indications for PP with G-CSFs in the clinical cases were not declared in about $2 \%$ of responses. In three-quarters (76\%) of responses, indications/lack of indications for PP administration were declared that were considered correct according to the guidelines and the experts' opinion. In clinical cases where indications for PP administration existed, $72 \%$ of the responses indicated that PP would be administered and 28\% indicated that PP would not be administered. In clinical cases where indications for PP administration were lacking, $25 \%$ of responses indicated that PP would be administered.

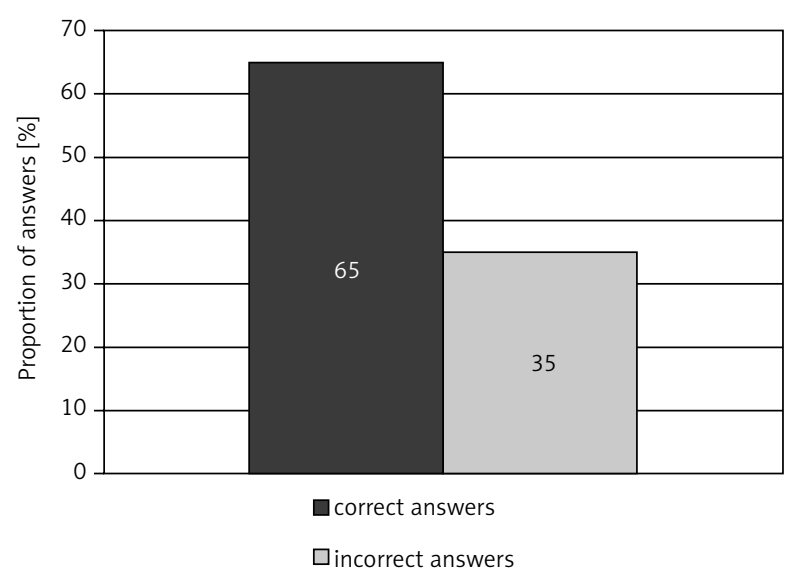

Fig. 2. Percentage of correct and incorrect answers concerning the risk of febrile neutropenia depending on the $\mathrm{CT}$ regimen

A higher percentage of correct answers were given in clinical cases where patients received a high-risk or low-risk regimen than in cases where patients received a moderate-risk regimen. In the breast cancer cases where a high-risk regimen was used, the correct answer to administer PP was given in over $85 \%$ of responses for the patients receiving AT and in $94 \%$ of responses for the patients receiving TAC (Fig. 3).

In cases with low-FN-risk regimens when there were no indications for use of PP, the correct response rate was $79 \%$ for palliative breast cancer, $85 \%$ for ovarian cancer, and $90 \%$ for lung cancer (Fig. 3).

Evaluation of indications for G-CSF PP was more problematic when the $C T$ regimen was associated with a moderate risk of FN. For example, FN risk in the R-CHOP regimen, used in patients with non-Hodgkin lymphoma (DLBCL and PMBL), is moderate according to the guidelines, but according to the experts' opinion, PP is indicated in the presence of additional risk factors, such as advanced age, comorbidities, female gender, bulky disease, superior vena cava syndrome (SVCS), poor general condition, possible tumour lysis syndrome with impaired renal function, and curative treatment (radical treatment). The average proportion of correct answers was approximately 54\%: $71 \%$ for DLBCL and 36\% for PMBL lymphoma (Fig. 3).

\section{Use of granulocyte colony-stimulating factors as primary prophylaxis}

Figure 4 shows the choice of G-CSF administration that would be given by participants, based on their assessment of the FN risk of the CT regimen. In over $90 \%$ of responses, G-CSF was not chosen as PP with low-risk CT regimens. For $\mathrm{CT}$ regimens with a high risk of $\mathrm{FN}$, about $90 \%$ of the responses stated that pegfilgrastim would be used on the second day of the cycle or G-CSF would be given daily for more than five days. This is consistent with the PTOK and EORTC guidelines. Pegfilgrastim was more frequently selected for PP than daily G-CSF.

\section{Discussion}

The results of the present study confirmed the high awareness among Polish oncologists of current PTOK and 


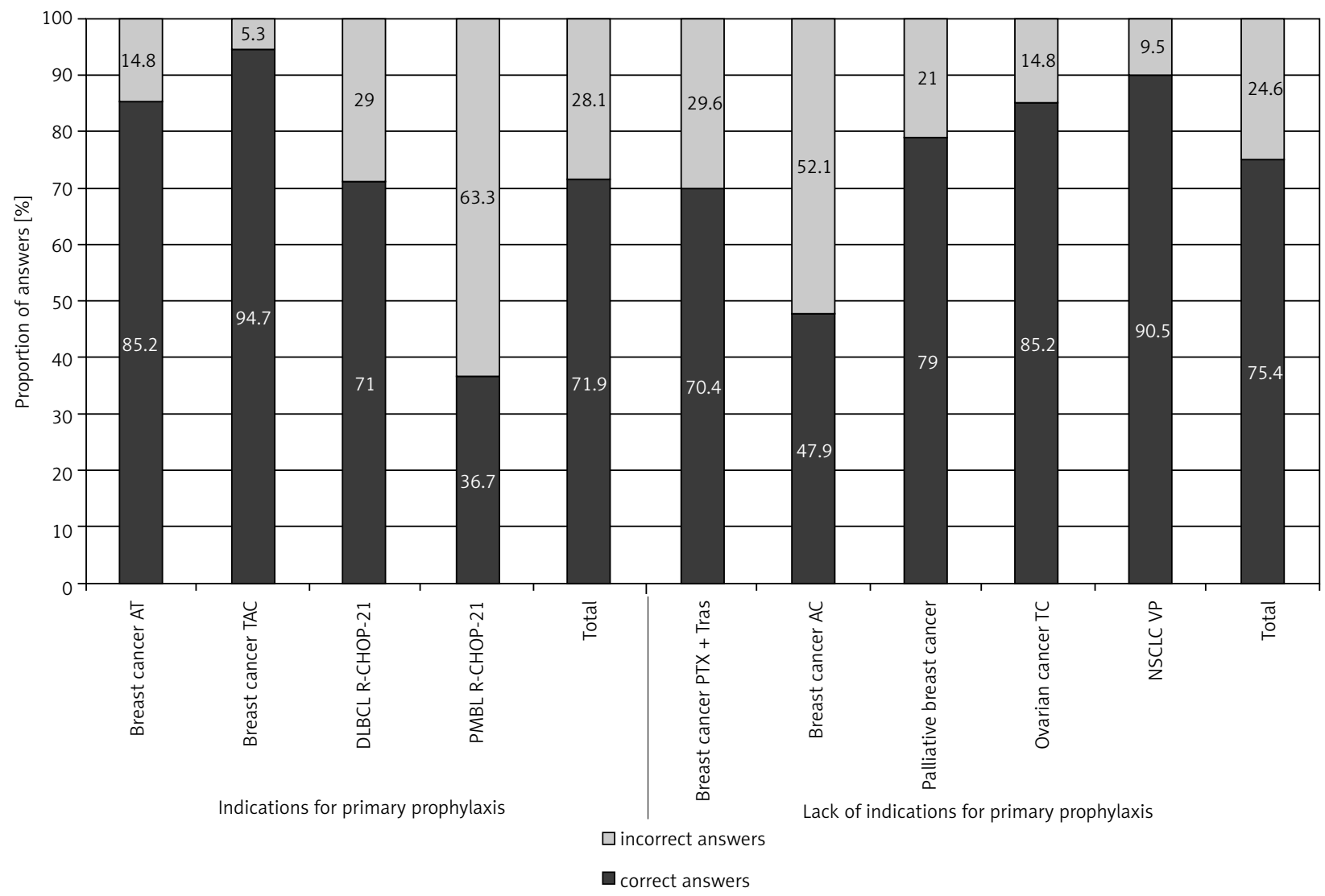

Note: Seven patient cases were assessed; in two them assessment of FN risk was performed twice: first due to CT change (docetaxel toxicity) and second due to disease progression (neoadjuvant AT in breast cancer then PTX + Tras after surgery and radiotherapy)

AT - doxorubicin, docetaxel; TAC - docetaxel, doxorubicin, cyclophosphamide; AC - doxorubicin, cyclophosphamide; DLBCL - diffuse large B-cell lymphoma; R-CHOP-21 - rituximab, cyclophosphamide, doxorubicin, vincristine, prednisone; PMBL - primary mediastinal B-cell lymphoma; PTX + Tras - paclitaxel + trastuzumab; AC - doxorubicin, cyclophosphamide; TC - paclitaxel, carboplatin; NSCLC - non-small-cell lung cancer; VP - vinorelbine, cisplatin

Fig. 3. Percentages of correct and incorrect answers to the question "Shall the patient be qualified for primary prophylaxis with G-CSF?" with reference to indications/lack of indications for primary prophylaxis based on Polish and European guidelines and experts' opinion

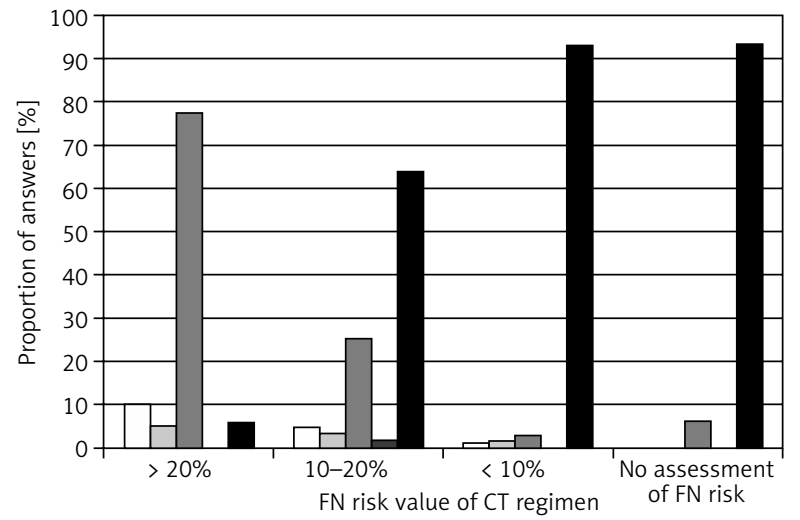

$\square$ Daily GCS-F longer than 5 days (> 5 days), s.c. from day 2 or 3 of CT cycle

$\square$ Daily GCS-F up to 5 days ( $<5$ days), s.c. from day 2 of CT cycle

$\square$ Pegfilgrastim s.c. on day 2 of CT cycle

$\square$ GCS-F administration would depend on the presence of severe neutropenia

- No G-CSF administration

Fig. 4. Answers provided to the question about G-CSF administration methods: "How would you administer G-CSF after the first cycle of CT in this situation?" with reference to the participant-assessed $\mathrm{CT}$ regimen $\mathrm{FN}$ risk
EORTC recommendations that FN risk should be assessed before each cycle of CT. Somewhat poorer awareness of the guidelines was observed for the evaluation of CT regimen FN risk by participants and with their recognition of indications or lack of indications for G-CSF PP.

Avoiding FN is important to improve patients' shortand long-term survival outcomes. According to Kuderer et al. [5], who analysed 14,000 patients hospitalised for FN in the years 1995-2000, the overall hospital mortality rate due to FN was $9.5 \%$ and was greater in the presence of concomitant diseases. In a more recent publication, Lyman et al. [6] reported that the adjusted mortality risk in patients with FN was at least $15 \%$ higher than matched patients without FN. Febrile neutropenia also leads to CT dose reductions and delays, resulting in lower $\mathrm{CT}$ relative dose intensity (RDI) that may in turn adversely affect long-term survival outcomes. Clinical studies in patients with breast cancer and NHL have shown that higher RDI levels are associated with improved long-term survival [7-10], and this relationship is supported by evidence from a prospective observational study involving over 2,500 patients in the early stages of various cancers, in which RDI $>85 \%$ was associated with considerably longer early dis- 
ease-free survival and overall survival than RDI $\leq 85 \%$ [7]. In addition, meta-analyses of clinical studies have found that G-CSF PP is associated with increased delivery of CT $[11,12]$ and reduced risk of mortality [12]. Therefore, the ability to correctly assess FN risk and the need for G-CSF is very important as it enables clinicians to optimise their approach to neutropenia management such that the risk of CT-limiting neutropenic complications are reduced and treatment outcomes are improved [6, 7].

The level of overall FN risk depends largely on the CT regimen used, and PP is recommended if the risk is more than $20 \%[2,4]$. When the risk is between $10 \%$ and $20 \%$, the use of PP is additionally based on patient-related factors such as age above 65 years, disease progression, poor general performance, malnutrition, female gender, low haemoglobin level $(<12 \mathrm{~g} / \mathrm{dl})$, liver and kidney dysfunction, and circulatory disease [2]. Although several studies have investigated the contribution of different patient-related risk factors to the overall FN risk [13], there is currently no validated model to quantify the FN risk of patients based on individual factors. The potential benefit of a standardised approach to individual FN risk factor-assessment was demonstrated in the current study: participants were better able to correctly identify the overall FN risk in patients receiving a clearly high- or low-risk regimen, but less concordance between the guidelines and the experts' advice was found in patients receiving moderate-risk regimens. These results suggest that an individual approach is taken to risk factor assessment, whereas a standardised approach may improve guideline adherence, particularly in patients receiving regimens with intermediate or undocumented FN risk.

The evaluation of $\mathrm{CT}$ regimen $\mathrm{FN}$ risk by participants was in agreement with the guidelines and the experts' opinion approximately two-thirds of the time, and greater agreement was observed with high-risk and moderate-risk regimens than with low-risk regimens. The reasons for this discrepancy are not clear but may relate to differences between reported FN rates in clinical studies and those observed in the participants' clinical practice. The guideline classifications of CT regimen risk are based predominantly on evidence from clinical studies, but neutropenia-related toxicities are frequently underreported in studies of patients with solid tumour and haematological malignancies $[14,15]$. For example, a phase 3 study of docetaxel plus cyclophosphamide in patients with breast cancer reported FN rates of 4-8\%, but subsequent publications from clinical practice experience reported rates in the range greater than $20 \%[16,17]$.

Accurate FN risk assessment is essential to guide appropriate use of G-CSF - this has implications both for clinical outcomes and healthcare resource utilisation [18]. In the current study, it appears that when the indications for PP were correctly recognised by participants, G-CSFs were usually administered in compliance with the national and international recommendations (daily G-CSF administered 24-72 hours after CT and continued daily for more than five days, or pegfilgrastim once-per-cycle on day two) (Fig. 4); when indications for PP were correctly identified, the incorrect method of G-CSF administration was chosen in only $5 \%$ of responses. The incorrect response most often referred to the administration of daily G-CSF, for a period shorter than five days. Shorter duration of prophylaxis with filgrastim has been shown to increase the hospitalisation risk in patients with breast cancer, lung cancer, or $\mathrm{NHL}$ [19]; each additional day of filgrastim administration reduced the risk of hospitalisation due to $\mathrm{FN}$ or infection by up to $23 \%$.

Pegfilgrastim was much more frequently selected for use in PP than daily G-CSF by participants, and it has been shown to be the same as or more effective in reducing the incidence of FN than daily G-CSF in randomised studies and in clinical practice [20-26], although recently a higher efficacy for pegfilgrastim was indicated in a meta-analysis of clinical studies [20] and in a Spanish observational study [25]. Administration of shorter courses of daily G-CSF than recommended by the guidelines have been observed in clinical practice, and the negative impact of this sub-optimal application of G-CSF was reported in a study by von Minckwitz et al. [27]. In this analysis of 11 clinical trials involving more than 2,000 breast cancer patients, the frequency of FN and of hospitalisations for FN and CT dose reductions in cycle 1 were significantly lower in patients receiving pegfilgrastim PP than those receiving current clinical practice G-CSF. The safety profiles of daily G-CSFs and pegfilgrastim are comparable [21-23]. From an economic perspective, while pegfilgrastim is more expensive than daily G-CSFs, PP with pegfilgrastim has been shown to be more cost-effective than daily G-CSFs in various settings [28, 29].

In summary, the GoPractice project allowed for an evaluation of clinical practice of Polish oncologists concerning the prevention of FN. The responses related to FN risk assessment and the method of PP administration indicated the need for periodic training to update and standardise knowledge in this field. We also recommend the introduction of readily available and clear supporting materials for physicians, which would enable quick and correct assessment of FN risk in oncological wards.

GoPractice was supported by Amgen Biotechnologia Sp. z 0.o. Editorial assistance was provided by James O'Kelly, an employee of Amgen Ltd.

\section{References}

1. Potemski P. Neutropenia. In: Zalecenia postępowania diagnostyczno-terapeutycznego w nowotworach złośliwych. Potemski P, Krzakowski M (red.). PTOK, Warszawa 2013; 537-547.

2. Aapro MS, Bohlius J, Cameron DA, et al.; European Organisation for Research and Treatment of Cancer. 2010 update of EORTC guidelines for the use of granulocyte- colony stimulating factor to reduce the incidence of chemotherapy-induced febrile neutropenia in adult patients with lymphoproliferative disorders and solid tumours. Eur J Cancer 2011; 47: 8-32.

3. Crawford J, Caserta C, Roila F; ESMO Guidelines Working Group. Hematopoietic growth factors: ESMO Clinical Practice Guidelines for the applications. Ann Oncol 2010; 21 Suppl 5: v248-51.

4. Smith TJ, Khatcheressian J, Lyman GH, et al. 2006 update of recommendations for the use of white blood cell growth factors: an evidence-based clinical practice guideline. J Clin Oncol 2006; 24: 3187-205. 
5. Kuderer NM, Dale DC, Crawford J, Cosler LE, Lyman GH. Mortality, morbidity, and cost associated with febrile neutropenia in adult cancer patients. Cancer 2006; 106: 2258-66.

6. Lyman GH, Kuderer NM, Crawford J, Wolff DA, Culakova E, Poniewierski MS, Dale DC. Predicting individual risk of neutropenic complications in patients receiving cancer chemotherapy. Cancer 2011; 117: 1917-27.

7. Lyman GH. Impact of chemotherapy dose intensity on cancer patient outcomes. J Natl Compr Canc Netw 2009; 7: 99-108.

8. Epelbaum R, Faraggi D, Ben-Arie Y, Ben-Shahar M, Haim N, Ron Y, Robinson E, Cohen Y. Survival of diffuse large cell lymphoma A multivariate analysis including dose intensity variables. Cancer 1990; 66: 1124-9.

9. Chirivella I, Bermejo B, Insa A, et al. Optimal delivery of anthracycline-based chemotherapy in the adjuvant setting improves outcome of breast cancer patients. Breast Cancer Res Treat 2009; 114: 479-84.

10. Bosly A, Bron D, Van Hoof A, et al. Achievement of optimal average relative dose intensity and correlation with survival in diffuse large B-cell lymphoma patients treated with CHOP. Ann Hematol 2008; 87: 277-83.

11. Kuderer NM, Dale DC, Crawford J, Lyman GH. Impact of primary prophylaxis with granulocyte colony-stimulating factor on febrile neutropenia and mortality in adult cancer patients receiving chemotherapy: a systematic review. J Clin Oncol 2007; 25: 3158-67.

12. Lyman GH, Dale DC, Culakova E, Poniewierski MS, Wolff DA, Kuderer NM, Huang M, Crawford J. The impact of the granulocyte colony-stimulating factor on chemotherapy dose intensity and cancer survival: a systematic review and meta-analysis of randomized controlled trials. Ann Oncol 2013; 24: 2475-84.

13. Lyman GH, Abella E, Pettengell R. Risk factors for febrile neutropenia among patients with cancer receiving chemotherapy: A sys tematic review. Crit Rev Oncol Hematol 2014; 90: 190-199.

14. Gregory SA, Abella S, Moore T. Underreporting of neutropenic toxicity associated with current treatment regimens for selected hematologic malignancies. Commun Oncol 2011; 8: 311-25.

15. Chan A, Verma S, Loibl S, et al. Reporting of myelotoxicity associated with emerging regimens for the treatment of selected solid tumors. Crit Rev Oncol Hematol 2012; 81: 136-50.

16. Jones S, Holmes FA, O'Shaughnessy J, et al. Docetaxel with cyclophosphamide is associated with an overall survival benefit com pared with doxorubicin and cyclophosphamide: 7-year follow-up of US Oncology Research Trial 9735. J Clin Oncol 2009; 27: 1177-83.

17. Vandenberg T, Younus J, Al-Khayyat S. Febrile neutropenia rates with adjuvant docetaxel and cyclophosphamide chemotherapy in early breast cancer: discrepancy between published reports and community practice - a retrospective analysis. Curr Oncol 2010; 17: 2-3.

18. Schnipper LE, Smith TJ, Raghavan D, Blayney DW, Ganz PA, Mulvey TM, Wollins DS. American Society of Clinical Oncology identifies five key opportunities to improve care and reduce costs: the top five list for oncology. J Clin Oncol 2012; 30: 1715-24.

19. Weycker D, Hackett J, Edelsberg JS, Oster G, Glass AG. Are shorter courses of filgrastim prophylaxis associated with increased risk of hospitalization? Ann Pharmacother 2006; 40: 402-7.

20. Cooper KL, Madan J, Whyte S, Stevenson MD, Akehurst RL. Granulocyte colony-stimulating factors for febrile neutropenia prophylaxis following chemotherapy: systematic review and meta-analysis. BMC Cancer 2011; 11: 404.

21. Green MD, Koelbl H, Baselga J, et al.; International Pegfilgrastim 749 Study Group. A randomized double-blind multicenter phase III study of fixed-dose single-administration pegfilgrastim versus daily filgrastim in patients receiving myelosuppressive chemotherapy. Ann Oncol 2003; 14: 29-35.

22. Holmes FA, Jones SE, O'Shaughnessy J, et al. Comparable efficacy and safety profiles of once-per-cycle pegfilgrastim and daily injection filgrastim in chemotherapy-induced neutropenia: a multicenter dose-finding study in women with breast cancer. Ann Oncol 2002; 13: 903-9.

23. Johnston E, Crawford J, Blackwell S, Bjurstrom T, Lockbaum P, Roskos L, Yang BB, Gardner S, Miller-Messana MA, Shoemaker D, Garst J, Schwab G. Randomized, dose-escalation study of SD/01 compared with daily filgrastim in patients receiving chemotherapy. J Clin Oncol 2000; 18: 2522-8.
24. Vogel CL, Wojtukiewicz MZ, Carroll RR, Tjulandin SA, Barajas-Figueroa LJ, Wiens BL, Neumann TA, Schwartzberg LS. First and subsequent cycle use of pegfilgrastim prevents febrile neutropenia in patients with breast cancer: a multicenter, double-blind, placebo-controlled phase III study. J Clin Oncol 2005; 23: 1178-84.

25. Almenar D, Mayans J, Juan O, et al. Pegfilgrastim and daily granulocyte colony-stimulating factor: patterns of use and neutropenia-related outcomes in cancer patients in Spain - results of the LEARN Study. Eur J Cancer Care (Engl) 2009; 18: 280-6.

26. Brugger W, Bacon P, Lawrinson S, Romieu G. Neutrophil recovery in elderly breast cancer patients receiving adjuvant anthracy zcline-containing chemotherapy with pegfilgrastim support. Crit Rev Oncol Hematol 2009; 72: 265-9.

27. von Minckwitz G, Schwenkglenks M, Skacel T, Lyman GH, Pousa AL, Bacon P, Easton V, Aapro MS. Febrile neutropenia and related complications in breast cancer patients receiving pegfilgrastim primary prophylaxis versus current practice neutropaenia management: results from an integrated analysis. Eur J Cancer 2009; 45: 608-17.

28. Hill G, Barron R, Fust K, Skornicki ME, Taylor DC, Weinstein MC, Lyman GH. Primary vs secondary prophylaxis with pegfilgrastim for the reduction of febrile neutropenia risk in patients receiving chemotherapy for non-Hodgkin's lymphoma: cost-effectiveness analyses. J Med Econ 2014; 17: 32-42.

29. Whyte S, Cooper KL, Stevenson MD, Madan J, Akehurst R. Cost-effectiveness of granulocyte colony-stimulating factor prophylaxis for febrile neutropenia in breast cancer in the United Kingdom. value Health 2011; 14: 465-74.

\section{Address for correspondence}

Prof. Marek Wojtukiewicz, MD, PhD

Department of Oncology

Medical University

Comprehensive Cancer Center

Bialystok, Poland

e-mail: mzwojtukiewicz@gmail.com

Submitted: 23.10 .2014

Accepted: $\quad 20.11 .2014$ 